\title{
Citocinas e quimiocinas no transplante renal
}

\author{
Cytokines and chemokines in renal transplantation
}

Autores

André Barreto

Pereira $^{1}$

Nilton Alves de

Rezende ${ }^{2}$

Antônio Lúcio

Teixeira Junior ${ }^{2}$

Mauro Martins

Teixeira $^{3}$

Ana Cristina Simões e Silva ${ }^{4}$

${ }^{1}$ Faculdade de Medicina da Universidade Federal de Minas Gerais (UFMG), Serviço de Nefrologia e Serviço de Transplante Renal da Santa Casa de Misericórdia de Belo Horizonte - Belo Horizonte, MG, Brasil

${ }^{2}$ Departamento de Clínica Médica da Faculdade de Medicina da UFMG - Belo Horizonte, MG, Brasil ${ }^{3}$ Departamento de Bioquímica e Imunologia do Instituto de Ciências Biológicas da UFMG - Belo Horizonte, MG, Brasil ${ }^{4}$ Departamento de Pediatria da Faculdade de Medicina da UFMG - Belo Horizonte, MG, Brasil

Data de submissão: 07/05/2009 Data de aprovação: 31/08/2009

\section{Correspondência para:} Ana Cristina Simões e Silva

Avenida Bernardo Monteiro, 1300 / apto 1104 Funcionários - Belo Horizonte - MG

CEP: 30150-281

E-mail: acssilva@hotmail. com

Declaramos a inexistência de conflitos de interesse.

\section{Resumo}

O transplante renal é a melhor modalidade de terapia renal substutiva até o momento. Infelizmente, a sobrevida do enxerto é interrompida pelos episódios de rejeição aguda ou mesmo de fibrose intersticial/atrofia tubular. A dosagem de quimiocinas e citocinas urinárias como ferramenta alternativa para o diagnóstico dessas complicações tem sido relatada nos últimos anos. Estas substâncias estão sabidamente relacionadas com os mecanismos imunoinflamatórios do transplante renal, podendo ser detectadas no tecido renal, no plasma e na urina de pacientes transplantados. Drogas anti-inflamatórias, inibidores do sistema renina angiotensina e alguns antagonistas de receptores de citocinas, ainda utilizados em nível experimental, podem interferir com a expressão desses mediadores do sistema imune e, por conseguinte, alterar a evolução do transplante renal. Neste sentido, pretende-se neste artigo fazer uma revisão dos estudos sobre a mensuração de citocinas/quimiocinas e dos seus receptores na urina, no plasma e no tecido renal de pacientes transplantados, no intuito de avaliar uma possível associação entre os níveis desses mediadores e as complicações do transplante renal e sobrevida do enxerto.

Palavras-chave: citocinas, quimiocinas, rejeição aguda, IF/TA, função renal.

[J Bras Nefrol 2009;31(4):286-296] CElsevier Editora Ltda.

\section{Abstract}

Renal transplantation is currently the best modality of renal substitutive therapy. Unfortunately graft survival is interrupted by episodes of acute rejection or even interstitial fibrosis/tubular atrophy. The measurement of urinary chemokines and cytokines as an alternative tool to diagnose these complications has been reported in past years. Those substances are clearly related to the immunoinflammatory mechanisms of renal transplantation, and can be detected in renal tissue, plasma, and urine samples of transplant recipients. Anti-inflammatory drugs, renin-angiotensin system inhibitors, and some antagonists of cytokines' receptors, although used only experimentally, can interfere with the expression of these immune system mediators and consequently alter renal transplantation outcome. This article reviewed studies on the measurement of cytokines/ chemokines and their receptors in urine, plasma, and renal tissue of transplant recipients, aiming at evaluating a possible association of the levels of those mediators with renal transplantation complications and allograft survival.

Keywords: cytokines, chemokines, acute rejection, IF/TA, renal function. 


\section{INTRODUÇÃO}

Vários estudos têm mostrado que a presença de rejeição aguda (RA) e a fibrose intersticial/atrofia tubular (IF/TA) são fatores importantes para a evolução do transplante renal. ${ }^{1,2}$ Embora a introdução de novas medicações imunossupressoras tenha propiciado melhor controle da RA, em torno de $10 \%$ dos pacientes transplantados ainda desenvolvem esta complicação no primeiro ano pós-transplante ${ }^{3}$ Além disso, em torno de 10 anos após o transplante, quase a totalidade dos pacientes apresenta IF/TA. ${ }^{4}$ Neste contexto, trabalhos recentes têm mostrado que algumas citocinas/ quimiocinas podem ser consideradas marcadores da RA e da IF/TA. ${ }^{5}$ Por meio de revisão sistemática da literatura, utilizando-se o software OVID no período de 1950 até a $4^{\text {a }}$ semana de janeiro de 2009, verificou-se grande variabilidade nos estudos referentes às citocinas e quimiocinas no transplante renal, desde a metodologia até os desfechos clínicos avaliados.

Dessa forma, este artigo de revisão tem por objetivo apresentar uma síntese da literatura atual com relação ao papel da mensuração de citocinas/quimiocinas e de seus receptores na urina, no plasma, no tecido renal de pacientes transplantados, no intuito de avaliar uma possível associação entre seus níveis e os eventos imunológicos do transplante renal, como a IF/TA, a RA e a sobrevida do rim transplantado. Serão também discutidos polimorfismos genéticos das citocinas e a relação dessas substâncias com o uso de drogas anti-inflamatórias e os processos infecciosos associados ao transplante renal.

\section{Citocinas e Quimiocinas - CONCEITO}

As citocinas são proteínas solúveis, de baixo peso molecular, produzidas geralmente em reposta ao estímulo antigênico, e que funcionam como um mensageiro químico para regulação do sistema imune adaptativo e inato. ${ }^{6}$ Se forem sintetizadas por fagócitos mononucleares, são denominadas monocinas e, quando produzidas por linfócitos, linfocinas. Por outro lado, existem evidências de que uma mesma proteína possa ser elaborada por linfócitos, monócitos e outras células, incluindo as células endoteliais. Historicamente, algumas citocinas foram denominadas interleucinas (IL), por serem produzidas por leucócitos e agir em outros leucócitos. Apesar de estudos posteriores mostrarem que as interleucinas podem atuar e ser produzidas por outras células além dos leucócitos, a nomenclatura foi mantida por questões de padronização. De fato, estas proteínas são produzidas por todas as células envolvidas na resposta e na apresentação de antígeno, especialmente pelos linfócitos T helper.
As quimiocinas são citocinas que apresentam papel central na fisiologia leucocitária, ao controlar o tráfego basal e inflamatório. A denominação quimiocina advém da propriedade de algumas citocinas em exercer quimiotaxia para leucócitos e outras células inflamatórias. Em outras palavras, as citocinas quimiotáticas passaram a ser chamadas de quimiocinas. ${ }^{7}$ As quimiocinas são polipeptídeos de 8 a $12 \mathrm{kD}$, com duas alças internas de dissulfeto, classificadas em famílias com base no número e na localização de resíduos de cisteínas N-terminais. As duas principais famílias são a CC, quimiocinas com resíduos de cisteína adjacentes, e família CXC, em que estes resíduos são separados por um aminoácido. ${ }^{7}$ As quimiocinas podem ser categorizadas em induzíveis e constitutivas. As induzíveis podem ser estimuladas por qualquer fator que altere a homeostase celular, e seu RNA mensageiro pode aumentar mais de 300 vezes em poucas horas de ativação. ${ }^{8}$ As constitutivas são responsáveis pelo tráfico leucocitário basal e pela formação da arquitetura de órgãos linfoides secundários. ${ }^{8}$

\section{Citocinas E OUIMIOCINAS NO TRANSPLANTE RENAL}

MECANISMOS IMUNOINFLAMATÓRIOS DO TRANSPLANTE RENAL

O transplante renal deve ser visto como um processo indutor de intensa reação inflamatória, onde um órgão proveniente do doador, repleto de antígenos imunogênicos, é apresentado ao complexo sistema imune do receptor. Em consequência, é esperado que as citocinas e quimiocinas tenham papel importante na mediação desse processo.

O papel das citocinas e quimiocinas no transplante renal é bastante complexo e os estudos mostram a participação tanto de mediadores relacionados com a produção de células T helper 1 (Th1) quanto de células T helper2 (Th2). Segundo Cardoni et al., ${ }^{5}$ na etapa de indução da resposta inflamatória do transplante renal, células apresentadoras de antígenos, linfócitos $\mathrm{T}$ ativados e células natural killers (NK), na presença de IL-12, IL-18 e fator de necrose tumoral alfa (TNFalfa) levam ao aumento de produção de células Th1. Consequentemente, ocorre aumento da expressão de genes de CD-26 e LAG-3 (genes de ativação linfocitária), e de receptores CCR5 (receptor de RANTES/ CCL5, MIP-1 $\alpha /$ CCL3 e MIP-1 $\beta / C C L 4)$ e CXCR3 (receptor de IP-10/CXCL10, I-TAC/CXCL11 e MIG/ CXCL9), expressos em células Th1 ativadas. Por outro lado, se estiverem presentes as IL-4, IL-10, IL-13, ocorre aumento da produção de células Th2 e estímulo da expressão de CD30, CD62L (cuja expressão 
promove recrutamento de órgãos linfoides periféricos), CCR3 (receptor de eotaxina em eosinófilos), CCR4 (receptor de TARC/CCL17, quimiocina do timo regulada por ativação), MDC/CCL22 (quimiocina derivada de macrófagos, expressa em células $\mathrm{T}$, NK e células dendríticas), CCR8 (receptor de I-309/ CCL1, derivado do gene 3 de ativação celular, que é expresso em células T e neutrófilos) e CXCR4 (receptor de SDF-1 $\alpha / \beta / C X C L 12$, que é um fator derivado do estroma expresso em células T-CD4 ativadas, células dendríticas e eosinófilos). ${ }^{5}$

De acordo com Hu e Knechtle, ${ }^{9}$ as quimiocinas podem influenciar pelo menos três aspectos da biologia do enxerto renal: 1) a restauração do fluxo sanguíneo no enxerto pode levar à lesão do tipo isquemia/reperfusão, na qual as quimiocinas recrutam leucócitos; 2) respostas do receptor à infecção durante a supressão imunológica envolvem as quimiocinas e 3) os componentes inflamatórios na RA e na IF/TA são controlados por quimiocinas.

\section{DROGAS IMUNOMODULADORAS}

Algumas drogas com ação imunomoduladora e antiinflamatória foram avaliadas no cenário do transplante renal. Grone et al. ${ }^{10}$ avaliaram a ação de um antagonista do receptor de RANTES/CCL5, o Met-RANTES, em ratos transplantados em uso de ciclosporina (Csa), sem uso de Csa, usando Csa e Met-RANTES, e sem uso de Csa mas em uso de Met-RANTES. Esses autores demonstraram que o antagonista do receptor de RANTES reduz a adesão dos leucócitos ao endotélio e a expressão de IL-1 $\alpha$, IL-1 $\beta$, TNF- $\alpha$, TNF- $\beta$ e IFN- $\delta .^{10}$ Avaliando, também, este mesmo antagonista, Song et al. ${ }^{11}$ demonstraram uma redução da infiltração de linfócitos e macrófagos em enxertos de ratos tratados com Csa após 2 semanas de transplante, além da redução da expressão de RNA mensageiro para IL-2, IL-1 $\beta$, TNF- $\alpha$ e RANTES/CCL5. Após 28 semanas de tratamento, houve redução da glomerulosclerose, da fibrose intersticial, da atrofia tubular, da proliferação intimal arterial e da infiltração de monócitos. ${ }^{11} \mathrm{O}$ Met-RANTES também reduziu a expressão de TGF- $\beta$ e PDGF-B (fator de crescimento derivado de plaquetas), apesar de não alterar a depuração de creatinina. ${ }^{11}$ Horuk et al. ${ }^{12}$ utilizaram um potente antagonista do receptor CCR1 (ligante de RANTES/CCL5, MIP-1 $\alpha$ / CCL3 e MCP-3/CCL7), o BX 471, em coelhos transplantados renais em uso de Csa, e demonstraram que este antagonista reduz a necrose do tecido renal, mas não é capaz de substituir o inibidor de calcineurina. $\mathrm{O}$ BX 471 está sendo apontado como um possível candidato para a terapêutica imunossupressora. ${ }^{12}$
Os inibidores de enzima conversora de angiotensina (IECA) são amplamente conhecidos como renoprotetores, em razão de suas ações antifibróticas e atividade no endotélio vascular. ${ }^{13,14}$ Avaliando biópsias renais de grupos de ratos transplantados em uso de IECA, Noris et al. ${ }^{15}$ demonstraram melhora da função renal e menor expressão de MCP-1/CCL2, mas não de RANTES/CCL5 e TGF- $\beta$ após 13 meses de acompanhamento.

As estatinas são drogas efetivas no tratamento das dislipidemias. Estudos têm demonstrado sua ação anti-inflamatória nos vasos sanguíneos, estabilizando placas de ateroma e melhorando a função endotelial. ${ }^{16}$ Wang et al. ${ }^{17}$ estudaram pacientes transplantados renais em uso de estatinas e mostraram redução dos níveis séricos de MCP-1/CCL2, RANTES/CCL5, CCR5 e CCR2 após o início da administração da droga.

Em resumo, o potencial terapêutico desses novos imunomoduladores e de fármacos que interferem com o sistema imunológico nos processos de RA e IF/TA ressalta a importância das citocinas e quimiocinas no transplante renal.

\section{Citocinas e quimiocinas no teCido Renal E NO SANGUE DE TRANSPLANTADOS RENAIS}

Várias quimiocinas e citocinas têm sido pesquisadas no sangue e no tecido renal de transplantados renais. ${ }^{5}$ Algumas dessas substâncias têm sido relacionadas com IF/TA, RA, presença de dislipidemia e infecções. ${ }^{5}$

$\mathrm{Na}$ prática clínica do transplante renal, a biópsia renal ainda é o exame de referência para o diagnóstico de RA ou IF/TA. ${ }^{18}$ Por outro lado, na avaliação do tecido renal, existe controvérsia quanto à predominância de quimiocinas e citocinas. Acredita-se que receptores, CCR5 e CXCR3, e seus ligantes apresentem maior expressão na RA. ${ }^{5}$

Robertson et al. ${ }^{19}$ mostraram maior expressão de MCP-1/CCL2 e MIP-1/CCL4 em rejeições agudas classificadas como BANFF 2 com relação aos casos BANFF 1, em um estudo de 47 biópsias renais com menos de 48 dias de transplante. Além disso, RANTES/ CCL5 e MIP-1 $\alpha /$ CCL3 tenderam ao mesmo comportamento, sem atingir, no entanto, diferença estatística na comparação entre os dois subgrupos. ${ }^{19}$ Por outro lado, Oliveira et al. ${ }^{20}$ não demonstraram aumento de expressão de MCP-1/CCL2, IL-8/CXCL8 e MIP-1 $\alpha /$ CCL3 em uma avaliação de biópsias renais com RA. Nos casos com RA, houve aumento de IL1-Ra e IL-6, enquanto MIP-1 $\alpha /$ CCL3 mostrou-se mais elevada na IF/TA. ${ }^{20}$ RANTES/CCL5 é uma quimiocina para células $\mathrm{T}$ de memória, monócitos e eosinófilos, que é expressa, durante a rejeição celular aguda, 3 a 5 dias após 
ativação das células T. Seu RNAm é detectado em células mononucleares infiltrativas e no epitélio tubular renal. ${ }^{21}$ RANTES/CCL5 é quimioatraente para linfócitos T, monócitos, células NK, basófilos e eosinófilos, além de ativar células inflamatórias. Por estes motivos, alguns autores consideram essa quimiocina um possível biomarcador da rejeição no transplante renal..$^{22}$ No entanto, Baer et al..$^{23}$ não demonstraram diferença nos níveis séricos e urinários de RANTES/CCL5 entre o $1^{\circ}$ e o $12^{\circ}$ dia de transplante renal.

Avaliando segmentos do enxerto, Ruster et al., ${ }^{24}$ em 2004, perceberam maior infiltração de células positivas para MCP-1/CCL2 e CCR5 (receptor celular de RANTES/CCL5, MIP-1 $\alpha /$ CCL3 e MIP-1ß/CCL4) na região tubulointersticial justacortical em 15 enxertos renais com RA, comparados a nove com IF/TA. Nestes últimos, houve maior expressão glomerular de MCP-1/CCL2 e tendência a menor infiltração generalizada de células positivas para RANTES/CCL5, MCP-1/CCL2, CCR2 e CCR5. ${ }^{24}$

Apesar da observação desses autores, Segerer et al. ${ }^{25}$ não encontraram diferença na expressão de MCP-1/CCL2 em 26 biópsias com rejeição aguda, com relação a cinco controles não transplantados, mas um aumento de CXCR3 (receptor celular de IP-10/CXCL10, MIG/CXCL9 e I-TAC/CXCL11) e CCR5. Em estudo anterior, este mesmo grupo de pesquisadores já havia demonstrado que IP-10/CXCL10 (que se liga ao receptor CXCR3), MIP-1 $\alpha / C C L 3$ e MIP-1 $\beta / C C L 4$ (que se ligam ao receptor CCR5) estavam aumentados em biópsias renais durante RA. ${ }^{26}$ Células inflamatórias infiltrativas, especialmente as células $\mathrm{T}$ e os macrófagos, expressaram os receptores de quimocinas, CCR5 e CXCR3. ${ }^{26}$

Outro estudo demonstrou, em biópsias de 16 pacientes transplantados, maior expressão de MIG/ CXCL9, CXCR3 e IP-10/CXCL10 naqueles com IF/ TA e glomerulopatia do transplante, com relação àqueles somente com IF/TA. ${ }^{27}$

A medida dos níveis plasmáticos pré-transplante destas citocinas e quimiocinas pode ser capaz de predizer a evolução para IF/TA e RA. Neste sentido, alguns estudos têm mostrado que a dosagem sérica prétransplante de IP-10/CXCL10 é capaz de predizer o risco para complicações no pós-transplante..$^{28-31}$

\section{CITOCINAS E QUIMIOCINAS NA URINA DE TRANSPLANTADOS RENAIS}

De modo geral, Hu \& Knechtle, ${ }^{9}$ avaliando as citocinas e quimiocinas urinárias em transplantados renais, propuseram a divisão desses marcadores em três grupos:
1) Citocinas/quimiocinas que estão aumentadas em receptores com RA, NTA (Necrose Tubular Aguda), IF/TA, função normal do enxerto, assim como indivíduos hígidos: angiogenina, TIMPs (tissues inhibitors of metalloproteinase) e receptor 2 solúvel de TNF- $\alpha$, ligante de receptor 3 indutor de apoptose relacionado ao TNF- $\alpha$.

2) Citocinas/quimiocinas que têm baixa expressão em receptores de transplante renal e indivíduos hígidos: IL-1beta, IL-2, sR $\alpha$, IL-6, MIP$1 \alpha / C C L 3$, MIP-1 $\beta / C C L 4$, MIP-3 $\alpha / C C L 20$, IL-18 e TNF- $\alpha$.

3) Citocinas/quimiocinas que estão mais aumentadas em receptores com RA, NTA e IF/TA do que naqueles com função normal do enxerto e indivíduos hígidos: Adiponectina, IGFBP-1 (proteína ligadora de fator de crescimento "insulina-like"-1), IGFBP-2, IGFBP-6, IL-8/CXCL8, leptina, MCP-1/CCL2, MIP-1 $\delta$, sTNFR1, osteoprotegerina e receptor ativador de uroquinase plasminogênio. ${ }^{9}$

Nesse sentido, Li et al..$^{32}$ mostraram um método para diagnóstico de RA em enxertos renais, mensurando RNA mensageiro de perforina e granzima $B$ em células de sedimento urinário com sensibilidade e especificidade maior que $80 \%$. Em artigo de revisão recente, foi sugerido que os compostos de NGAL (lipocalina associada à gelatinase de neutrófilos humanos) e cistatina $\mathrm{C}$, para um painel plasmático, e NGAL, IL-18 (citocina induzida e clivada em túbulo proximal) e KIM-1 (molécula de lesão renal 1, expressa em células tubulares renais proximais), para um painel urinário, podem ser biomarcadores de lesão renal aguda. ${ }^{33}$

Em estudo com amostras de urina de 80 pacientes, Woltman et al..$^{34}$ detectaram que MIP-3 $\alpha /$ CCL20 está aumentado na RA, correlacionando-se com os níveis de creatinina. No entanto, tal elevação não precede a alteração de função renal. ${ }^{34}$

Outros trabalhos tentaram relacionar a excreção urinária de TGF- $\beta$ com a rejeição crônica do rim transplantado. Yamada et al., ${ }^{35}$ avaliando 16 pacientes com função renal estável após 1 ano de transplante, associaram o aumento dos níveis urinários de TGF- $\beta$ e da atividade de renina plasmática à perda da função renal. Neste estudo, sete pacientes com IF/ TA apresentaram maior excreção urinária de TGF- $\beta$ e atividade de renina plasmática, em resposta a inibidores de enzima conversora de angiotensina, do que aqueles sem IF/TA. ${ }^{35}$ Teppo et al. ${ }^{36}$ avaliaram 79 receptores, 6 meses após o transplante renal, e verificaram que o aumento da excreção urinária de TGF- $\beta$ 
está relacionado com a lesão tubular proximal e com a piora da função renal. Matl et al.$^{37}$ observaram em biópsias renais que a expressão tecidual de TGF- $\beta$ estava relacionada com a vasculopatia crônica, mas não com a presença de IF/TA. Avaliando polimorfismos genéticos para citocinas, Tinckam et al. ${ }^{38}$ e Alakulppi et al. ${ }^{39}$ verificaram que determinados polimorfismos para o gene do TGF- $\beta$ apresentam risco aumentado de RA. De acordo com Citterio et al. ${ }^{40}$ o uso de diferentes imunossupressores tem influência na expressão de TGF- $\beta$ sanguíneo. Portanto, a determinação das concentrações sérica e urinária de TGF- $\beta$ tem mostrado resultados promissores, correlacionando-se bem com o padrão de evolução da rejeição de enxerto renal. Existe a perspectiva de que as dosagens sanguínea e urinária de TGF- $\beta$ possam ser instrumentos importantes na abordagem do transplante renal, principalmente no que diz respeito à intervenção terapêutica precoce da RA e ao seu prognóstico.

A Tabela 1 resume os principais estudos sobre citocinas/quimiocinas urinárias em transplantados renais.

\begin{tabular}{|c|c|c|c|c|c|c|c|c|}
\hline Tabela 1 & \multicolumn{8}{|c|}{$\begin{array}{l}\text { RESUMO DAS PRINCIPAIS PUBLICAÇÕES SOBRE CITOCINAS/OUIMIOCINAS URINÁRIAS } \\
\text { EM TRANSPLANTADOS RENAIS }\end{array}$} \\
\hline Autor & Ref & $\mathrm{N}$ & Citocina & VPP & VPN & Sens. & Espec. & Objetivo \\
\hline Prodjosudjadi & 49 & 20 & MCP-1 & $100 \%$ & $84 \%$ & $64 \%$ & $100 \%$ & RA \\
\hline Paolo & 46 & 29 & EGF, IL-6 & NA & NA & NA & NA & RA e NIA \\
\hline Grandaliano & 50 & 20 & MCP-1 & NA & NA & NA & NA & RA \\
\hline Sibbring & 60 & 27 & IL-8, MIP-1 $\beta$ & NA & NA & NA & NA & RA \\
\hline Smith & 47 & 13 & IL-2, IL-6, IL 8, IL-10, TNF & NA & NA & NA & NA & RA, ITU \\
\hline Yamada & 35 & 16 & TGF & $83 \%$ & $78 \%$ & $71 \%$ & $88 \%$ & $\mathrm{FE}$ \\
\hline \multirow[t]{3}{*}{$\mathrm{Hu}$} & 54 & 99 & $\mathrm{IP}-10+\mathrm{MIG}$ & $86,6 \%$ & $88,9 \%$ & $88,6 \%$ & $87 \%$ & RA, BK e NTA \\
\hline & & & MIG & $92,1 \%$ & $82,7 \%$ & $79,5 \%$ & $93,5 \%$ & \\
\hline & & & IP-10 & $90,5 \%$ & $87,5 \%$ & $86,4 \%$ & $91,3 \%$ & \\
\hline Kanmaz & 55 & $7 *$ & MIG, IP-10 & NA & NA & NA & NA & RA \\
\hline Kotsch & 59 & 26 & $\begin{array}{l}\text { RANTES, IL-10, IL-2, } \\
\text { TGF, TNF, IFN }\end{array}$ & NA & NA & NA & NA & RA \\
\hline \multirow[t]{2}{*}{ Tatapudi } & 58 & 58 & IP-10, & NA & NA & $100 \%$ & $78 \%$ & RA \\
\hline & & & CXCR3 & NA & NA & $63 \%$ & $83 \%$ & \\
\hline Teppo & 36 & 79 & TGF & NA & NA & NA & NA & $\mathrm{FE}$ \\
\hline Woltman & 34 & 80 & $\mathrm{MIP}-3 \alpha$ & NA & NA & NA & NA & RA \\
\hline \multirow[t]{4}{*}{ Jimenez } & 48 & 19 & IL-4 & $100 \%$ & $77 \%$ & $66 \%$ & $100 \%$ & RA \\
\hline & & & IL-5 & $100 \%$ & $71 \%$ & $55 \%$ & $100 \%$ & RA \\
\hline & & & IL-10 & $100 \%$ & $90 \%$ & $88 \%$ & $100 \%$ & RA \\
\hline & & & IFN- $\gamma$ & $100 \%$ & $83 \%$ & $77 \%$ & $100 \%$ & RA \\
\hline \multirow[t]{2}{*}{ Hauser } & 56 & 54 & MIG & $70 \%$ & $97 \%$ & $93,3 \%$ & $89 \%$ & RA \\
\hline & & & IP-10 & $81,8 \%$ & $89 \%$ & $60 \%$ & $96,2 \%$ & RA \\
\hline Baer & 23 & 17 & RANTES & NA & NA & NA & NA & Proteinúria \\
\hline Sadeghi & 75 & 66 & IL-6, IL-8, sIL-1RA & NA & NA & NA & NA & Bacteriúria assintomática \\
\hline Matz & 57 & 35 & IP-10 & NA & NA & $58 \%$ & $75 \%$ & FE \\
\hline Ciszek & 74 & 47 & IL-6, IL-8 & NA & NA & NA & NA & Bacteriúria assintomática \\
\hline Boratynska & 76 & 17 & MCP-1 & NA & NA & NA & NA & Decoy cells \\
\hline Helantera & 77 & 46 & TGF- $\beta$, I-CAM- 1 , TNF- $\alpha$ & NA & NA & NA & NA & CMV \\
\hline
\end{tabular}

RA: rejeição aguda; FE: função do enxerto; CMV: citomegalovirus; NA: não se aplica;

BK: poliomavirus; NIA: nefrite intersticial aguda; NTA: necrose tubular aguda; Ref: referência bibliográfica.

VPP: valor preditivo positivo; VPN: valor preditivo negativo; ITU: infecção do trato urinário. 
O fator de crescimento epidermoide (EGF) é um peptídeo promotor de crescimento, que se encontra expresso na alça de Henle e no túbulo contorcido distal. ${ }^{41-44}$ Este peptídeo é sintetizado quase exclusivamente dentro do rim, e sua concentração se reduz dramaticamente em pacientes com insuficiência renal aguda..$^{45}$ Paolo et al.$^{46}$ avaliaram níveis urinários e a expressão em biópsia renal de EGF e IL-6 em 29 pacientes transplantados renais, aos 7 e 21 dias de transplante, e antes do tratamento de rejeição. Três transplantados renais com função renal estável e 10 amostras de tecidos viáveis de rins com carcinoma foram usados como controle. Dos pacientes transplantados, 16 apresentavam RA e 13 NTA ou nefrotoxicidade por ciclosporina. O EGF estava reduzido nessas situações. Já a IL-6 apresentouse aumentada na RA, mas inalterada na NTA ou nefrotoxicidade por ciclosporina ${ }^{46}$ Smith et al.${ }^{47}$ detectaram aumento de IL-6, IL-8/CXCL8 e TNF- $\alpha$ em amostras únicas de urina de três transplantados renais com RA em um grupo de 13 pacientes. Jimenez et al. ${ }^{48}$ relataram que pacientes que desenvolvem rejeição ao enxerto renal apresentam níveis mais elevados de IL-10/CXCL10 e IFN- $\gamma$ no plasma e na urina, em comparação com pacientes sem rejeição. Estes autores acreditam que as citocinas e quimiocinas podemse originar tanto de células inflamatórias infiltrativas quanto de células renais. ${ }^{48}$

Prodjosudjadi et al., na Holanda, avaliaram o nível de MCP-1/CCL2 em plasma e urina de 24 horas de 20 pacientes com RA e em 20 pacientes sem rejeição. Foi possível correlacionar o nível urinário desta citocina com o grau de rejeição, demonstrando seu aumento no grupo que apresentava rejeição mais acentuada. ${ }^{49}$ Em urina de nove horas, Grandaliano et al., avaliando 13 transplantados renais com RA e sete com NTA, detectaram aumento da expressão de MCP-1/CCL2 nos pacientes com RA em comparação aos casos de NTA. ${ }^{50}$

Níveis urinários elevados de TGF- $\beta$ foram encontrados no período pós-transplante inicial. Os níveis elevados dos primeiros dias se reduzem gradualmente em um período de 2 meses, mantendo-se, entretanto, maiores do que em indivíduos hígidos. ${ }^{51}$ Os níveis de TGF- $\beta$ têm sido correlacionados com excreção urinária de albumina e de $\alpha 1$-microglobulina 6 meses após o transplante renal. ${ }^{36}$ A elevação urinária do TGF- $\beta$ tem sido associada também ao grau de fibrose intersticial na IF/TA. ${ }^{52,53}$

Hu et al. ${ }^{54}$ analisando amostras únicas de urina pré-biópsia renal de 99 pacientes, demonstraram elevação de I-TAC/CXCL11, MIG/CXCL9 e IP-10/
CXCL10 em vigência de RA, nefropatia por poliomavírus e NTA, mas não em casos de rejeição borderline, IF/TA e função estável de enxerto. MIG/CXCL9 e IP-10/CXCL10 apresentaram níveis mais elevados, com sensibilidade de $88,6 \%$ e especificidade de $87 \%$ para diagnóstico das complicações mencionadas. ${ }^{54}$ Ressalta-se ainda que, com o tratamento da RA, os níveis urinários de MIG/CXCL9 e IP-10/CXCL10 se reduziram dias antes da diminuição da creatinina sanguínea. IP-10/CXCL10 urinária se reduziu em todos os pacientes 2 a 5 dias antes da diminuição da creatinina sérica. Estes resultados indicam que a elevação de níveis urinários de quimiocinas ligadoras a CXCR3 se correlaciona bem com a disfunção do enxerto, e a determinação destas quimiocinas na urina pode ser útil para monitorar a efetividade da terapia antirrejeição, em conjunto com a medida da creatinina. ${ }^{54} \mathrm{Kanmaz}$ et al.${ }^{55}$ mostraram que os níveis urinários de MIG/CXCL9 e IP-10/ CXCL10 de sete macacos transplantados renais aumentaram antes da elevação da creatinina na presença de RA. Nas biópsias renais, houve aumento da expressão de MIG/CXCL9 em glomérulos, túbulos e células infiltrativas. ${ }^{55}$ Hauser et al. ${ }^{56}$ verificaram, em 15 pacientes com RA dentro de um grupo de 69 transplantados renais, que a dosagem de MIG/CXCL9 e IP-10/CXCL10 em amostras únicas de urina permitia a predição de RA, assim como de resposta ao tratamento. MIG/CXCL9 apresentou maior sensibilidade $(93,3 \%)$ e especificidade $(89 \%)$ para RA, com boa correlação com IP-10/CXCL10 $(\mathrm{p}<0,01)$, e não se alterou em presença de NTA. ${ }^{56}$ $\mathrm{O}$ aumento dessas citocinas ocorreu de 2 a 3 dias antes do aumento da creatinina e 6 a 7 dias antes do diagnóstico histopatológico de RA. ${ }^{56}$ Matz et al. ${ }^{57}$ acompanharam 35 transplantados renais por 6 meses e mostraram que a medida do RNA mensageiro para IP-10/CXCL10 urinário no primeiro mês pós-transplante apresentava sensibilidade de $58 \%$ e especificidade de $75 \%$ para predizer a função renal medida 3 e 6 meses após o transplante. Sensibilidade de $71 \%$ e especificidade de $95 \%$ para o diagnóstico de RA foram atribuídas à dosagem urinária de IP-10/CXCL10 2 a 3 dias antes da biópsia renal. ${ }^{57}$ Tatapudi et al..$^{58}$ sugerem que a mensuração do RNA mensageiro para o IP-10/CXCL10 ou para o receptor de quimiocina CXCR3 em células urinárias pode tornar-se uma forma não invasiva de elucidar o tráfego celular durante os episódios de RA em enxertos renais humanos. Esses autores 
obtiveram 63 espécimes de urina de 58 transplantados submetidos à biópsia renal e 27 amostras urinárias de 24 indivíduos sem alteração de função renal. ${ }^{58}$ Foi detectado que o nível de RNA mensageiro para o IP-10/CXCL10 ou CXCR3 prediz o tráfego celular intraenxerto, relacionando-se com a RA. ${ }^{58}$

Resultados referentes ao aumento da expressão do RANTES/CCL5 urinário em presença de RA têm sido relatados por alguns autores. ${ }^{21-24,59}$ Baer et al..$^{23}$ avaliaram amostras de urina de 24 horas e sangue de 17 transplantados renais com e sem RA nos primeiros 12 dias de transplante renal. Esses autores não detectaram diferença estatisticamente significativa, apesar de haver aumento dos níveis de RANTES/ CCL5 com relação ao grupo-controle. ${ }^{23}$ Entretanto, encontraram correlação da proteinúria $(r=0,83$, $\mathrm{p}=0,0001)$ e da albuminúria $(\mathrm{r}=0,727, \mathrm{p}=0,0062)$ dos 17 pacientes com o nível de RANTES/CCL5 urinário, além de redução significativa destes valores entre o $1^{\circ}$ e o $12^{\circ}$ dia de transplante. ${ }^{23}$ Neste mesmo estudo, também foram dosados os níveis séricos desta quimiocina, sem que fossem detectadas diferenças ou correlação com os níveis urinários ${ }^{23}$ Kotsch et al. ${ }^{59}$ estudaram principalmente a expressão urinária de RNA mensageiro para RANTES/ CCL5, CD3 e granulosina em 14 pacientes transplantados renais com RA comprovada por biópsia renal. Corroborando os achados de Baer et al., ${ }^{23}$ esses autores detectaram aumento de granulosina associado à elevação de RANTES e CD3 em, respectivamente, oito e cinco pacientes, no momento do diagnóstico da rejeição. ${ }^{59}$

$\mathrm{Na}$ avaliação das citocinas e quimiocinas urinárias na vigência da RA, tomados em conjunto, os trabalhos relatados mostraram elevações dos níveis urinários de MIP-3 $\alpha /$ CCL20, IL-8/CXCL8, IL-6, TNF, IL-10，IFN，MCP-1/CCL2，IP10/CXCL10，MIG/ CXCL9， I-TAC/CXCL11,RANTES/CCL..$^{59,24,60,46-}$ 50,75,54-56,59,16 Somente MIP-1ß/CCL4 ${ }^{60}$ urinária não se mostrou aumentada, enquanto o EGF apresentou redução. ${ }^{46}$ Como preditores de complicações e alterações futuras da função renal, os níveis de TGF- $\beta$ e IP-10/CXCL10 apresentaram associação à função renal de 6 meses e 4 anos após o transplante. ${ }^{35,36}$ IP-10/CXCL10, MIG/CXCL9, CXCR3, RANTES/ CCL5 e o percentual de ligação de IL-2 relacionaramse com a ocorrência de RA. ${ }^{47,56,58}$ IP-10/CXCL10 e MIG/CXCL9 também foram considerados úteis como preditores da resposta ao tratamento da RA. ${ }^{9,54,56}$

\section{POLIMORFISMO GENÉTICO DAS QUIMIOCINAS}

\section{E CITOCINAS}

No estudo de polimorfismos genéticos de citocinas/ quimiocinas e seus receptores, verificam-se resultados conflitantes com relação à ocorrência de RA e IF/TA. Apesar disso, alguns trabalhos mostraram as seguintes associações:

1) Estudos demonstraram que aqueles pacientes com polimorfismos genéticos que determinam aumento da produção de TGF- $\beta$ apresentam menos episódios de rejeição, ${ }^{38}$ sugerindo um papel imunossupressor e de regulação da resposta imune para o TGF- $\beta$.

2) RA apresentou associação a alguns polimorfismos e alterações genotípicas de diversas citocinas, tais como MCP-1/CCL2 (2518 GG), CCR2 (V64I), CCR5, TNF- $\alpha$ (-308 A/A), TGF- $\beta$ (T/C，G/G，10TT/25GG，10CC/25GG)，IFN- $\gamma$ (+874 T/T, T/A), IL-10 (ATA/ATA, ACCACC, GCCATA), IL-2(T/G), IL-1Ra (genótipo com ale$\operatorname{los} 1 / 2)$ e IL-18 (-137GG). ${ }^{38,39,61-67}$

3) IF/TA foi associada ao genótipo $308 \mathrm{~A} / \mathrm{A}$ de TNF- $\alpha$ de pacientes com mismatches em DRB e ao genótipo 10 T/T de TGF- $\beta .^{67}$

4) Alguns polimorfismos genéticos de MCP-1/ CCL2 (-2518 G) se mostraram associados à função do enxerto a longo prazo. ${ }^{65}$

5) No doador renal, o genótipo 308 G/A do TNF- $\alpha$ influenciou na ocorrência de função tardia do enxerto e se relacionou com a IF/TA, assim como o polimorfismo $174 \mathrm{C} / \mathrm{C}$ de IL-6. ${ }^{39,68}$

6) $\mathrm{O}$ polimorfismo $-403 \mathrm{G} / \mathrm{A}$ e $\ln 1.1 \mathrm{~T} / \mathrm{C}$ de RANTES/CCL5 apresentou associação somente com a recorrência de RA. ${ }^{69}$

7) Os polimorfismos genéticos de IL-1 $\alpha$ (-889), IL-1 $\beta$ (-511 e +3962), IL-1R (PstI 970), IL-4 (-590), IL-4R (1902) e IL-6 (+3247) não apresentaram relação com a ocorrência de RA. Os genótipos estudados para TNF- $\alpha(+488,-238,-308)$, TGF- $\beta(-800,-509$ e +915 cod 25$)$, e IFN- $\gamma(+874)$ também não tiveram alteração significativa na vigência de IF/TA ou RA, nem mesmo associação com a função do enxerto renal. Polimorfismos genéticos de CCR2 (V64I) e RANTES/CCL5 (-403, -109 e -28) não apresentaram correlação com IF/ TA ou função do enxerto renal. ${ }^{70-71}$

A Tabela 2 resume os principais estudos sobre polimorfismo genético de citocinas/quimiocinas em transplantados renais. 


\begin{tabular}{|c|c|c|c|c|c|}
\hline Tabela 2 & $\begin{array}{l}\text { RESUMO DA } \\
\text { EM TRANSPI }\end{array}$ & $\begin{array}{l}\text { RINCIP } \\
\text { CADOS }\end{array}$ & $\begin{array}{l}\text { JBLICAÇÕES SOBRE POL } \\
\text { IS }\end{array}$ & DRFISMO GENÉTICO DE CITOCINA & UIMIOCINAS \\
\hline Autor & Ref & $\mathrm{N}$ & Citocina & Alelo & Objetivo \\
\hline \multirow[t]{2}{*}{ Kruger } & 64 & 232 & MCP-1/CCL2 & 2518 GG & FE \\
\hline & & & CCR2 & V64l & FE \\
\hline \multirow[t]{2}{*}{ Abdi } & 62 & 163 & CCR2 & V64I & RA \\
\hline & & & CCR5 & 59029 G/G ou A/G & RA \\
\hline \multirow[t]{4}{*}{ Mc Daniel } & 65 & 77 & TGF- $\beta$ & $\mathrm{T} / \mathrm{C}, \mathrm{G} / \mathrm{G}$ & RA, FE \\
\hline & & & IFN- $\gamma$ & +874 T/T, T/A & RA, FE \\
\hline & & & IL-10 & ATA/ATA & RA, FE \\
\hline & & & IL-2 & $T / G$ & RA \\
\hline \multirow[t]{4}{*}{ Alakulppi } & 39 & 206 & TNF- $\alpha$ doador & $-308 \mathrm{G} / \mathrm{A}$ do doador & RA \\
\hline & & 291 & TNF- $\alpha$ & $-308 \mathrm{~A} / \mathrm{A}$ & RA \\
\hline & & & TGF- $\beta$ & $10 T T / 25 G G$ e $10 \mathrm{CC} / 25 \mathrm{GG}$ & RA \\
\hline & & & IL-10 & ATA/ATA, ACCACC e GCCATA & RA \\
\hline \multirow[t]{3}{*}{ Nikolova } & 68 & 66 & TGF- $\beta$ & $10 T T$ & IF/TA \\
\hline & & 28 & TNF- $\alpha$ doador & -308 G/A do doador & IF/TA \\
\hline & & & IL-6 & $174 \mathrm{C} / \mathrm{C}$ do doador & $\mathrm{IF} / \mathrm{TA}$ \\
\hline Tinckam & 38 & 118 & TGF- $\beta$ & $25 \mathrm{G} / \mathrm{G}$ & RA (protetor) \\
\hline Manchanda & 66 & 136 & IL-1Ra & Intron $2-1 / 2$ & RA \\
\hline \multirow{2}{*}{ Kang } & 61 & 167 & MCP-1/CCL2 & 2518 GG & RA tardia \\
\hline & & & MCP-1/CCL2 + CCR2 & $2518 G G+V 64 I G G$ & RA e RA tardia \\
\hline Kruger & 69 & 261 & RANTES/CCL5 & $-403 \mathrm{G} / \mathrm{A}, \ln 1.1 \mathrm{~T} / \mathrm{C}$ & RA recorrente \\
\hline Singh & 63 & 296 & CCR2 & $+/ 641$ & RA (protetor) \\
\hline \multirow[t]{2}{*}{ Kim } & 67 & 226 & IL-18 & $-137 G G$ & RA \\
\hline & & & TNF- $\alpha$ & $308 \mathrm{~A} / \mathrm{A}$ & FE \\
\hline
\end{tabular}

RA: rejeição aguda; FE: função do enxerto; IF/TA: atrofia tubular/fibrose intersticial;

Ref: referência bibliográfica.

\section{Citocinas, QUIMIOCINAS E INFECÇÕES}

Considerando-se a elevada frequência e o caráter imunomodulador da infecção pelo CMV, Nordoy et al. ${ }^{72}$ avaliaram 10 pacientes com doença por este vírus, cinco com antigenemia positiva, mas sem doença, e $10 \mathrm{com}$ antigenemia negativa e sem doença. Esses autores correlacionaram a antigenemia de CMV com os níveis séricos de IL-8/CXCL8 ( $\mathrm{r}=0,63)$ e MCP-1/ CCL2 $(r=0,44)$ durante o pico da doença ou período de positividade, e com o IL-8/CXCL8 $(r=0,57)$, durante o seguimento da infecção. MIP-1 $\alpha /$ CCL3 não apresentou correlação com antigenemia ou doença por $\mathrm{CMV}^{72}$ Em estudo recente em biópsias renais de ratos transplantados com e sem esta infecção, Soule et al. ${ }^{73}$ demonstraram que os ratos infectados desenvolviam IF/TA mais precocemente, além de apresentarem maior expressão de RANTES/CCL5, MCP-1/CCL2, MIP-1 $\alpha / C C L 3$, e IP-10/CXCL10. RANTES/CCL5 e MCP-1/CCL2 ao final do período de acompanhamento, e IP-10/CXCL10 no início do processo.
Na bacteriúria assintomática, foi verificada maior excreção de IL-8/CXCL8, IL-6, e sIL-1Ra. ${ }^{74-75}$ Durante infecções virais oportunistas, MCP-1/CCL2 apresentou maior excreção urinária relacionada à doença causada por poliomavírus, ${ }^{76}$ e o TGF- $\beta$ urinário se elevou na infecção por $\mathrm{CMV}^{77}$

Analisando 22 transplantados renais com bacteriúria assintomática (17 mulheres e cinco homens) e 25 (18 mulheres e sete homens) sem estas alterações infecciosas, foi demonstrado que a IL-8/CXCL8 em amostra única de urina está aumentada nos transplantados e que não há diferença nos níveis de IL-6. ${ }^{74} \mathrm{Em}$ amostras de urina de 24 horas, na ausência de infecção urinária ou infecção por citomegalovirus (CMV), Sibbring et al. ${ }^{60}$ detectaram aumento de IL-8/CXCL8 em 21 pacientes com RA celular, com relação a seis com rejeição vascular crônica, mas sem alterações imuno-histoquímicas sugestivas de rejeição nas biópsias renais. A produção de MIP-1ß/CCL4 urinário não diferiu entre os grupos estudados. ${ }^{60}$ 
A expressão desta citocina nos túbulos renais contorcidos e coletores, assim como a expressão de IL-8/ CXCL8, foram maiores do que a de MCP-1/CCL2. ${ }^{60}$

Sadeghi et al..$^{75}$ avaliaram dois grupos de $66 \mathrm{pa}$ cientes com e sem bacteriúria assintomática, 12 a 21 meses após o transplante renal. Em análise de amostra única de urina e plasma verificaram que os homens com bacteriúria apresentavam maior nível de IL-6, IL-8/CXCL8, antagonista do receptor solúvel de IL-1 (sIL-1Ra) e razão urina/plasma de IL-6 do que aqueles sem bacteriúria. ${ }^{75}$ Entre as mulheres, os níveis de IL-6 e IL-8/CXCL8 foram similares, com leve aumento dos níveis sIL-1Ra e da razão urina/plasma de IL-6. ${ }^{75}$

Ilkka et al. ${ }^{77}$ verificaram maior excreção urinária de TGF- $\beta$ em transplantados renais na vigência de infecção por CMV. Em outra avaliação de infecção oportunista em 174 transplantados renais em um período de 5 anos, Boratynska et al. ${ }^{76}$ mostraram aumento de MCP-1/CCL2 urinário em 17 pacientes que apresentavam "decoy cells" em amostras únicas de urina com relação aos controles. ${ }^{76}$

Para diferenciar RA de ITU, Smith et al. mostraram que as concentrações de IL-2 e IL-2B são 3,6 a 2,8 vezes maiores, respectivamente, em RA que em ITU. ${ }^{47}$ De acordo com Hauser et al., os níveis urinários de MCP-1 na RA podem ser afetados pela presença de ITU, o que não aconteceria com MIG. ${ }^{56}$

\section{Conclusão}

Este artigo de revisão mostra que as quimiocinas e citocinas, sobretudo urinárias, podem refletir e predizer a função do enxerto nos meses subsequentes à sua mensuração e, algumas vezes, suas concentrações se elevam alguns dias antes da ocorrência de rejeição. Além disso, algumas dessas substâncias podem ser úteis na detecção de processos infecciosos nos pacientes transplantados. No entanto, é importante ressaltar que os resultados dos estudos ainda são controversos e não permitem a recomendação de mensurar quimiocinas e citocinas e/ou determinar polimorfismos genéticos durante o seguimento clínico dos transplantados renais. Por outro lado, analisando os principais achados em conjunto, acredita-se que não apenas uma substância seja importante na avaliação dos pacientes transplantados, mas um conjunto delas, que poderiam vir a ser utilizadas para uma predição não invasiva e mais eficaz do estado do enxerto renal, comportandose como mais uma ferramenta ou mesmo um biomarcador no controle dos transplantados renais.

\section{RefERÊNCIAS}

1. Tesi RJ, Elkhammas EA, Henry ML, Davies EA, Salazar A, Ferguson RM. Acute rejection episodes, best predictor of long-term primary cadaveric renal transplant survival. Transplant Proc 1993; 25:901-2.

2. Jevnikar AM, Mannon RB. Late Kidney Allograft Loss: What We Know about It, and What We Can Do about It. Clin J Am Soc Nephrol 2008; 3:S56-67.

3. www.usrds.org Acessado em abril 2008.

4. Nankivell BJ, Borrows RJ, Fung CLS, O'Connell PJ, Allen RDM, Chapman JR. The Natural History of Chronic Allograft Nephropathy. N Engl J Med 2003; 349:2326-33.

5. Cardoni RL, Prigoshin N, Tambutti ML, Ferraris JR. Regulatory cytoquines in the response to the allogeneic renal transplant. Medicina 2005; 65:54-62.

6. Dallman MJ. Cytokines and transplantation: Th1/ Th2 regulation of the immune response to solid organ transplants in the adult. Curr Opin Immunol 1995; 7:632-8.

7. Abbas AK, Lichtman AH. Cytokines. Cellular and Molecular Immunology. 5th Edition. Philadelphia: Saunders, Elsevier Science, 2003, pp. 243-74.

8. Gerard C, Rollins BJ. Chemokines and disease. Nat Immunol 2001; 2:108-15.

9. $\mathrm{Hu} \mathrm{H}$, Knetchle SJ. Elevation of multiple cytokines/chemokines in urine of human renal transplant recipients with acute and chronic injuries: potential usage for diagnosis and monitoring. Transpl Rev 2006; 20:16571.

10. Grone H-J, Weber C, Weber KSC et al. Met-RANTES reduces vascular and tubular damage during acute renal transplant rejection: blocking monocyte arrest and recruitment. FASEB J 1999; 13:1371-83.

11. Song E, Zou H, Yao Y et al. Early application of MetRANTES ameliorates chronic allograft nephropathy. Kidney Int 2002; 61:676-85.

12. Horuk R, Shurey S, Ng HP et al. CCR1-specific nonpeptide antagonist: efficacy in a rabbit allograft rejection model. Immunol Let 2001; 76:193-201.

13. Remuzzi G, Perico N. Protecting single-kidney allografts from long-term functional deterioration. J Am Soc Nephrol 1998; 9:1321-32.

14. Taal MW, Brenner BM. Renoprotective benefits of RAS inhibition: from ACEI to angiotensin II antagonists. Kidney Int 2000; 57:1803-17.

15. Noris M, Mister M, Pezzotta A et al. ACE inhibition limits chronic injury of kidney transplant even with treatment started when lesions are established. Kidney Int 2003; 64: 2253-61.

16. Sorrentino S, Landmesser U. Nonlipid-lowering Effects of Statins. Curr Treat Options Cardiovasc Med 2005; 7:459-66.

17. Wang J, Zou H, Li Q, Wang Y, Xu Q. The expression of monocyte chemoattractant protein-1 and C-C chemokine receptor 2 in post-kidney transplant patients and the influence of simvastatin treatment. Clinica Chimica Acta 2006; 373:44-8.

18. Silva DM, Garcia JP, Ribeiro AR et al. Utility of biopsy in kidney transplants with delayed graft function and acute dysfunction. Transplant Proc 2007; 39:376-7. 
19. Robertson H, Morley A, Talbot D, Callanan K, Kirby J. Renal allograft rejection: $\beta$-chemokine involvement in the development of tubulitis. Transplantation 2000; 69:684-7.

20. Oliveira JGG, Xavier P, Neto S, Mendes AA, Guerra LER. Monocytes-macrophages and cytokines/chemokines in fine-needle aspiration biopsy cultures: enhances interleukin-1 receptor antagonist synthesis in rejectionfree kidney transplant patients. Clin Transpl 1997; 63:1751-6.

21. Pattison J, Nelson PJ, Huie P et al. RANTES chemokine expression in cell mediated transplant rejection of the kidney. Lancet 1994; 343:209-11.

22. Krensky AM, Ahn Y-T. Mechanism of Disease: regulation of RANTES (CCL5) in renal disease. Nature Clin Pract 2007; 3:164-70.

23. Baer PC, Koziolek M, Fierlbeck W, Geiger H. CCchemokine RANTES is increased in serum and urine in the early post-transplantation period of human renal allograft recipients. Kidney Blood Press Res 2005; 28:48-54

24. Ruster M, Sperschneider H, Funfstuck R, Stein G, Grone H-J. Differential expression of $\beta$-chemokines MCP-1 and RANTES and their receptors CCR1, CCR2, CCR5 in acute rejection and chronic allograft nephropathy of human renal allografts. Clin Nephrol 2004; 61:30-9.

25. Segerer S, Cui Y, Eitner F et al. Expression of chemokines and chemokine receptors during human renal transplant rejection. Am J Kidney Dis 2001; 37:518-31.

26. Segerer S, Mack M, Regele H, Kerjaschki D, Schlöndorff D. Expression of the C-C chemokine receptor 5 in human kidney diseases. Kidney Int 1999; 56:52-64.

27. Akalin E, Dikman S, Murphy B, Bromberg JS, Hancock. Glomerular infiltration by CXCR3+ ICOS+ activated T cells in chronic allograft nephropathy with transplant glomerulopathy. Am J Transplant 2003; 3:1116-20.

28. Romagnani P. From basic science to clinical practice: use of cytokines and chemokines as therapeutic targets in renal diseases. J Nephrol 2005; 18:229-33.

29. Rotondi M, Rosati A, Buonamano A et al. High pretransplant serum levels of CXCL10/IP-10 are related to increased risk of renal allograft failure. Am J Transplant 2004; 4:1466-74.

30. Dufour JH, Dziejman M, Liu MT, Leung JH, Lane TE, Luster AD. IFN-gamma-inducible protein 10 (IP10; CXCL10)-deficient mice reveal a role for IP-10 in effector $\mathrm{T}$ cell generation and trafficking. J Immunol 2002; 168:3195-204.

31. Wang X, Yue TL, Ohlstein EH, Sung CP, Feuerstein GZ. Interferon-inducible protein 10 involves vascular smooth muscle cell migration, proliferation, and inflammatory response. J Biol Chem 1996; 271:24286-93.

32. Li BG, Hartono C, Ding R et al. Noninvasive diagnosis of renal-allograft rejection by measurement of messenger RNA for perforin and granzyme B in urine. $\mathrm{N}$ Engl J Med 2001; 344:947-54.

33. Devarajan P. Emerging Biomarkers of Acute Kidney Injury. Contrib Nephrol. Basel Karger 2007; 156:20312.

34. Woltman AM, Fijter JW, van der Kooij SW et al. MIP$3 \alpha /$ CCL20 in renal transplantation and its possible involvement as dendritic cell chemoattractant in allograft rejection. Am J Transplant 2005; 5:2114-25.
35. Yamada K, Hatakeyama E, Arita S, Sakamotu K, Kashiwabara H, Hamaguchi K. Prediction of chronic renal allograft dysfunction from evaluation of TGF $\beta-1$ and the renin-angiotensin system. Clin Exp Nephrol 2003; 7:238-42.

36. Teppo AM, Honkanen E, Finne P, Tornroth T, Gronhagen-Riska C. Increased urinary excretion of alpha1-microglobulin at 6 months after transplantation is associated with urinary excretion of transforming growth factor-beta 1 and indicates poor long-term renal outcome. Transplantation 2004; 78:719-24.

37. Matl I, Viklicky O, Voska L, Lodererova A, Vitko S. The effect of different immunosuppressive regimens on TGF-beta1 expression in kidney transplant patients. Transpl Int. 2005; 18:668-71.

38. Tinckam K, Rush D, Hutchinson I et al. The Relative Importance of Cytokine Gene Polymorphisms in the Development of Early and Late Acute Rejection and Six-Month Renal Allograft Pathology. Transplantation 2005; 79:836-41.

39. Alakulppi NS, Kyllönen LE, Jäntti VT et al. Cytokine Gene Polymorphisms and Risks of Acute Rejection and Delayed Graft Function after Kidney Transplantation. Transplantation 2004; 78:1422-8.

40. Citterio F, Pozzetto U, Romagnoli J et al. Plasma levels of Transforming Growth Factor- $\beta 1$ in Renal Transplant Recipients Receiving Different Immunosuppressive Regimens. Transplant Proc 2004; 36:698-9.

41. Rall LB, Scott J, Bell GI et al. Mouse prepro-epiderma growth factor synthesis by the kidney and other tissues. Nature 1985; 313:228-31.

42. Salido EC, Yen PH, Shapiro LJ, Fisher DA, Barajas L. In situ hybridization of pre-pro epidermal growth factor mRNA in the mouse kidney. Am J Physiol 1989; 256:F632-8.

43. Nouwen EJ, De Broe ME. EGF and TGF-beta in the human kidney: identification of octopal cells in the collecting duct. Kidney Int 1994; 45:1510-21.

44. Gesualdo L, Di Paolo S, Calabro A et al. Expression of epidermal growth factor and its receptor in normal and diseased human kidney. An immunohistochemical and in situ hybridization study. Kidney Int 1996; 49:65665 .

45. Taira T, Yoshimura A, Iizuka K, Iwasaki S, Ideura T, Koshikawa S. Urinary epidermal growth factor levels in patients with acute renal failure. Am J Kidney Dis 1993; 22:656-61.

46. Di Paolo S, Gesualdo L, Stallone G, Ranieri E, Schena FP. Renal expression and urinary concentration of EGF and IL-6 in acutely dysfunctioning kidney transplanted patients. Nephrol Dial Transplant 1997; 12:2687-93.

47. Smith SD, Wheeler MA, Lorber MI, Weiss RM. Temporal changes of cytokines and nitric oxide products in urine from renal transplant patients. Kidney Int 2000; 58:829-37.

48. Jimenez R, Ramírez R, Carracedo J et al. Cytometric bead array (CBA) for the measurement of cytokines in urine and plasma of patients undergoing renal rejection. Cytokine 2005; 32:45-50.

49. Prodjosudjadi W, Daha MR, Gerritsma JSJ et al. Increased urinary excretion of monocyte chemoattractant protein-1 during acute renal allograft rejection. Nephrol Dial Transplant 1996; 11:1096-103. 
50. Grandaliano G, Gesualdo L, Ranieri E, Monno R, Stallone G, Schena FP. Monocyte chemotactic peptide-1 expression and monocyte infiltration in acute renal transplant rejection. Transplantation 1997; 63:414-20.

51. Goumenos DS, Tsakas S, Savidaki I et al. Urinary transforming growth factor (TGF- $\beta 1$ ) excretion in renal allograft recipients during the early post-transplantation period. Ren Fail 2003; 25:561-8.

52. Boratynska M. Urine excretion of transforming growth factor-beta 1 in chronic allograft nephropathy. Ann Transplant 1999; 4:23-8.

53. Grandaliano G, Di Paolo S, Monno R et al. Proteaseactivated receptor 1 and plasminogen activator inhibitor 1 expression in chronic allograft nephropathy. Transplantation 2001; 72:1437-43.

54. $\mathrm{Hu} \mathrm{H}$, Aizenstein BD, Puchalski A, Burmania JA, Hamawy MM, Knechtle SJ. Elevation of CXCR3binding chemokines in urine indicates acute renal-allograft dysfunction. Am J Transplant 2004; 4:432-7.

55. Kanmaz T, Feng P, Torrealba J et al. Surveillance of acute rejection in baboon renal transplantation by elevation of interferon-gamma inducible protein-10 and monokine induced by interferon-gamma in urine. Transplantation 2004; 78:1002-7.

56. Hauser IA, Spiegler S, Kiss E et al. Prediction of acute renal allograft rejection by urinary monokine induced by IFN-gamma (MIG). J Am Soc Nephrol 2005; 16:1849-58.

57. Matz M, Byer J, Wunsch D et al. Early post-transplant urinary IP-10 expression after kidney transplantation is predictive of short- and long-term graft function. Kidney Int 2006; 69:1683-90.

58. Tatapudi RR, Muthukumar T, Dadhania D et al. Noninvasive detection of renal allograft inflammation by measurements of mRNA for IP-10 and CXCR3 in urine. Kidney Int 2004; 65:2390-7.

59. Kotsch K, Mashreghi MF, Bold G et al. Enhanced granulysin mRNA expression in urinary sediment in early and delayed acute renal allograft rejection. Transplantation. 2004; 77:1866-75.

60. Sibbring JS, Sharma A, McDicken IW, Sells RA, Christmas SE. Localization of C-X-C and C-C chemokines to renal tubular epithelial cells in human kidney transplants is not confined to acute cellular rejection. Transplant Immunol 1998; 6:203-8.

61. Kang SW, Park SJ, Kim YW et al. Association of MCP-1 and CCR2 polymorphisms with the risk of late acute rejection after renal transplantation in Korean patients. Int J Immunogenet 2008; 35:25-31.

62. Abdi R, Tran TB, Sahagun-Ruiz A et al. Chemokine receptor polymorphism and risk of acute rejection in human renal transplantation. J Am Soc Nephrol 2002; 13:754-8

63. Singh R, Kapoor R, Srivastava A, Mittal RD. Impact of chemokine receptor CCR2 and CCR5 gene polymorphism on allograft outcome in North Indian renal transplant recipients. Scand J Immunol 2009; 69:51-6.

64. Kruger B, Schroppel B, Ashkan R et al. A monocyte chemoattractant protein-1 (MCP-1) polymorphism and outcome after renal transplantation. J Am Soc Nephrol 2002; 13:2585-9.
65. McDaniel DO, Barber WH, Nguyan C et al. Combined analysis of cytokine genotype polymorphism and the level of expression with allograft function in AfricanAmerican renal transplant patients. Transpl Immunol. 2003; 11:107-19.

66. Manchanda PK, Bid HK, Kumar A, Mittal RD. Genetic association of interleukin-1beta and receptor antagonist (IL-1Ra) gene polymorphism with allograft function in renal transplant patients. Transpl Immunol 2006; 15:289-96.

67. Kim CD, Ryu HM, Choi JY et al. Association of G-137C IL-18 promoter polymorphism with acute allograft rejection in renal transplant recipients. Transplantation 2008; 86:1610-44.

68. Nikolova PN, Ivanova MI, Mihailova SM et al. Cytokine gene polymorphism in kidney transplantation - impact of TGF-beta 1, TNF-alpha and IL-6 on graft outcome. Transpl Immunol 2008; 18:344-8.

69. Kruger B, Borger CA, Obed A et al. RANTES/CCL5 polymorphism as a risk factor for recurrent acute rejection. Clin Transplant 2007; 21:385-90.

70. Marshall SE, McLaren AJ, Haldar NA, Bunce M, Morris PJ, Welsh KI. The impact of recipient cytokine genotype on acute rejection after renal transplantation. Transplantation. 2000; 70:1485-91.

71. Brabcova I, Petrasek J, Hribova P et al. Genetic variability of major inflammatory mediators has no impact on the outcome of kidney transplantation. Transplantation 2007; 84:1037-44.

72. Nordøy I, Müller F, Nordal KP, Rollag H, Aukrust P, Frøland SS. Chemokines and soluble adhesion molecules in renal transplant recipients with cytomegalovirus infection. Clin Exp Immunol 2000; 120:333-7.

73. Soule JL, Streblow DN, Andoh TF, Kreklywich CN, Orloff SL. Cytomegalovirus accelerates chronic allograft nephropathy in a rat renal transplant model with associated provocative chemokine profiles. Transplant Proc 2006; 38:3214-20.

74. Ciszek M, Paczek L, Bartlomiejczyk I, Mucha K. Urine Cytokines Profile in Renal Transplant Patients with Asymptomatic Bacteriuria. Transplantation 2006; 81:1653-7.

75. Sadeghi M, Daniel V, Naujokat C et al. Strong inflammatory cytokine response in male and strong anti-inflamatory response em female kidney transplant recipients with urinary tract infection. Transplant Intern 2005; 18:177-85.

76. Boratynska M, Dubinski B, Rybka K, Jezior D, Szyber P, Klinger M. Imunocytological Urinalysis and Monocyte Chemotactic Peptide-1 in Renal Tranpslant Recipients With Polyomavirus Replication. Transplantation Proceedings. 2006; 38:151-4.

77. Helantera I, Teppo A-M, Koskinen P, Törnroth T, Grönhagen-Riska C, Lautenschlager I. Increased urinary excretion of transforming growth factor- $\beta 1$ in renal transplant recipients during cytomegalovirus infection. Transplant Immunol 2006; 15:217-21. 